\title{
Comparison of Clinical Outcomes of BRCA1/2 Pathologic Mutation, Variants of Unknown Significance, or Wild Type Epithelial Ovarian Cancer Patients
}

\section{Kyung Jin Eoh, $\mathrm{MD}^{1}$ \\ Hyung Seok Park, MD2,3 \\ Ji Soo Park, MD, PhD2,4 \\ Seung-Tae Lee, MD, $\mathrm{PhD}^{2,5}$ \\ Jeongwoo Han, MD, PhD2,6 \\ Jung-Yun Lee, MD, PhD 1 \\ Sang Wun Kim, MD, PhD ${ }^{1}$ \\ Sunghoon Kim, MD, PhD \\ Young Tae Kim, MD, PhD ${ }^{1}$ \\ Eun Ji Nam, MD, PhD',2}

\section{Purpose}

The purpose of this study was to investigate the clinical features of epithelial ovarian cancer (EOC) patients according to BRCA1/2 mutation status (mutation, variant of uncertain significance [VUS], or wild type).

\section{Materials and Methods}

We analyzed 116 patients whose BRCA1/2 genetic test results were available for mutation type and clinical features, including progression-free survival (PFS), overall survival (OS), and response rate. These characteristics were compared according to BRCA1/2 mutation status.

\section{Results}

Thirty-seven (37/116, 31.9\%) BRCA1/2 mutations were identified (BRCA1, 30; BRCA2, 7). Mutation of c.3627_3628insA (p.Leu1209_Glu1210?fs) in BRCA1 was observed in five patients (5/37, 13.5\%). Twenty-five patients had BRCA1/2 VUSs (25/116, 21.6\%). Personal histories of breast cancer were observed in $48.6 \%$ of patients with BRCA1/2 mutation (18/37), $16.0 \%$ of patients with BRCA1/2 VUS (4/25), and $7.4 \%$ of patients with BRCA wild type $(4 / 54)(p<0.001)$. Patients with BRCA1/2 mutation showed longer OS than those with $B R C A 1 / 2$ wild type $(p=0.005)$. No significant differences were detected in PFS, OS, or response rates between patients with $B R C A 1 / 2$ VUS and BRCA1/2 mutation $(p=0.772$, $\mathrm{p}=0.459$, and $\mathrm{p}=0.898$, respectively).

\section{Conclusion}

Patients with $B R C A 1 / 2$ mutation had longer OS than those with BRCA1/2 wild type. Patients with BRCA1/2 mutation and BRCA1/2 VUS displayed similar prognoses.

\section{Key words}

Ovarian epithelial cancer, BRCA1, BRCA2, Prognosis
Correspondence: Eun Ji Nam, MD, PhD Department of Obstetrics and Gynecology, Institute of Women's Life Medical Science, Women's Cancer Clinic, Yonsei University College of Medicine, 50-1 Yonsei-ro, Seodaemun-gu, Seoul 03722, Korea Tel: $82-2-2228-2250$

Fax: 82-2-313-8350

E-mail: NAHMEJ6@yuhs.ac

Received March 29, 2016

Accepted July 8, 2016

Published Online July 27, 2016 


\section{Introduction}

Epit helial ovarian cancer (EOC) remains the gynecological cancer with the highest mortality because over two-thirds of patients have advanced disease upon diagnosis [1]. Only $10 \%$ of cases are due to an inherited predisposition, while the majority are attributable to alterations in $B R C A 1$ and $B R C A 2$, which present the strongest risk factors for breast and ovarian cancers [2]. Indeed, studies of lifetime risk of ovarian cancer have revealed that it ranges from $39 \%$ to $54 \%$ in women identified as having a $B R C A 1 / 2$ mutation, with lower risk associated with $B R C A 2$ mutation than BRCA1 [3].

Most patients with $B R C A 1 / 2$ mutations have a demonstrably more favorable outcome than those with sporadic ovarian cancers $[4,5]$. However, the mechanism of this purported survival advantage conferred by BRCA1/2 mutation is not entirely clear. Some have speculated that it results from a greater susceptibility to chemotherapy owing to a significantly higher growth fraction in BRCA1/2 -associated malignancies [6]. However, others have not been able to confirm the significant survival advantage conferred by BRCA1/2 mutations, specifically in terms of long term overall survival (OS) $[7,8]$. Indeed, poorer survival for patients with $B R C A 1 / 2$ mutations has been reported $[9,10]$, although it remains unclear why different investigators have noted such varying effects of $B R C A 1 / 2$ alterations on survival.

Several previous studies of $B R C A 1 / 2$ and EOC have been conducted in Korea, and BRCA1/2 mutations were found in $33 \%$ of patients with a strong family history of EOC $[11,12]$. Possible candidates of a founder mutation in Korea have also been reported [13]. Moreover, a large, prospective, nationwide study of Korean breast cancer patients and their $B R C A 1 / 2$ status suggested that BRCA1 mutation has a signif- icant negative impact on survival [14]. However, only a few studies have analyzed the effects of $B R C A 1 / 2$ mutation status on the clinical prognosis of EOC patients of Asian ethnicity [15]. Furthermore, clinical features of EOC patients who have a $B R C A 1 / 2$ variant of uncertain significance (VUS), which is a gene mutation that has an unknown effect on protein function, have not been reported to date [16]. The potential for an alternate interpretation of a BRCA1/2 VUS over time carries with it possible disparate clinical implications. Thus, characterization of patients with $B R C A 1 / 2$ VUS is required for counseling and follow-up.

Therefore, this study was conducted to investigate clinical features of EOC patients according to BRCA1/2 mutation status (BRCA1/2 mutation vs. BRCA1/2 VUS vs. BRCA1/2 wild type), including survival.

\section{Materials and Methods}

\section{Patient selection and pathologic review}

The study protocol was approved by the Institutional Review Board. During the review of medical records, we obtained data describing several patient characteristics, including age at diagnosis, histologic type, and surgical stage as classified by the International Federation of Gynecology and Obstetrics criteria.

A patient selection diagram is shown in Fig. 1. A total of 711 patients were pathologically confirmed to have EOC from January 1999 to May 2015. Of these, 595 had uninformative $B R C A 1 / 2$ mutation statuses and were therefore excluded. A BRCA1/2 genetic test was performed for the

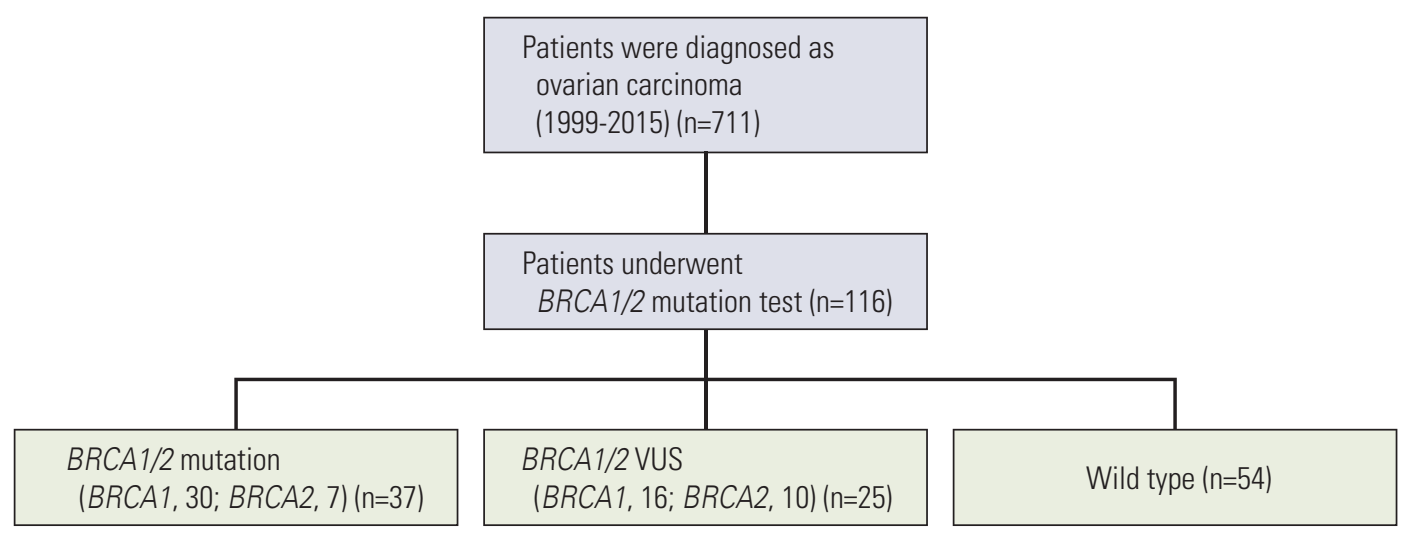

Fig. 1. Patient selection diagram. VUS, variant of uncertain significance. 
Table 1. Patient characteristics

\begin{tabular}{|c|c|c|c|c|c|}
\hline Characteristic & $\begin{array}{c}\text { Overall } \\
\text { population }(n=116)\end{array}$ & $\begin{array}{c}B R C A 1 / 2 \\
\text { mutation }(n=37)\end{array}$ & $\begin{array}{c}\text { BRCA1/2 } \\
\text { VUS (n=25) }\end{array}$ & $\begin{array}{c}B R C A 1 / 2 \\
\text { wild type }(n=54)\end{array}$ & p-value \\
\hline \multicolumn{6}{|l|}{ Age } \\
\hline Mean \pm SD & $52.2 \pm 11.4$ & $52.4 \pm 9.3$ & $52.0 \pm 11.6$ & $52.2 \pm 12.8$ & $0.99^{\text {a) }}$ \\
\hline \multicolumn{6}{|l|}{ Stage FIGO } \\
\hline I-II & $21(18.1)$ & $2(5.4)$ & $6(24.0)$ & $13(24.1)$ & $0.059^{b)}$ \\
\hline III-IV & $94(81.0)$ & 34 (91.9) & $19(76.0)$ & $41(75.9)$ & \\
\hline NI & $1(0.9)$ & $1(2.7)$ & 0 & 0 & \\
\hline \multicolumn{6}{|l|}{ Histology } \\
\hline Serous & $91(78.4)$ & $32(86.5)$ & $18(72.0)$ & $41(75.9)$ & $0.198^{\mathrm{b})}$ \\
\hline Mucinous & $8(6.9)$ & 0 & $2(8.0)$ & $6(11.1)$ & \\
\hline Endometrioid & $5(4.3)$ & 0 & $2(8.0)$ & $3(5.6)$ & \\
\hline Clear cells & $5(4.3)$ & 0 & $1(4.0)$ & $4(7.4)$ & \\
\hline Others & $3(2.6)$ & $2(5.4)$ & $1(4.0)$ & 0 & \\
\hline NI & $4(3.4)$ & $3(8.1)$ & $1(4.0)$ & 0 & \\
\hline \multicolumn{6}{|l|}{ Grade } \\
\hline $02-1$ & $45(38.8)$ & $9(24.3)$ & $10(40.0)$ & $26(48.1)$ & $0.260^{b)}$ \\
\hline 3 & $60(51.7)$ & $23(62.2)$ & $14(56.0)$ & $23(42.6)$ & \\
\hline NI & $11(9.5)$ & $5(13.5)$ & $1(4.0)$ & $5(9.3)$ & \\
\hline \multicolumn{6}{|l|}{ Optimal surgery } \\
\hline Yes & $82(70.7)$ & $22(59.5)$ & $20(80.0)$ & $40(74.1)$ & $0.130^{\mathrm{b})}$ \\
\hline No & $22(19.0)$ & $11(29.7)$ & 0 & $11(20.4)$ & \\
\hline NI & $12(10.3)$ & $4(10.8)$ & $5(20.0)$ & $3(5.6)$ & \\
\hline \multicolumn{6}{|c|}{ Personal history of breast cancer } \\
\hline Yes & $26(22.4)$ & $18(48.6)$ & $4(16.0)$ & $4(7.4)$ & $<0.001^{\mathrm{b})}$ \\
\hline No & $89(76.7)$ & $18(48.6)$ & $21(84.0)$ & $50(92.6)$ & \\
\hline NI & $1(0.9)$ & $1(2.7)$ & 0 & 0 & \\
\hline \multicolumn{6}{|c|}{ Family history of breast/ovarian cancer } \\
\hline Yes & $47(40.5)$ & $18(48.6)$ & $9(36.0)$ & $20(37.0)$ & $\left.0.299^{b}\right)$ \\
\hline No & $65(56.0)$ & $16(43.2)$ & $15(60.0)$ & $34(63.0)$ & \\
\hline NI & $4(3.4)$ & $3(8.1)$ & $1(4.0)$ & 0 & \\
\hline
\end{tabular}

Values are presented as number (\%). VUS, variant of uncertain significance; SD, standard deviation; FIGO, International Federation of Gynecology and Obstetrics; NI, not indicated. ${ }^{\text {a) }}$ ANOVA test, ${ }^{\text {b) }}$ Chi-square Pearson's test.

remaining 116 patients. A gynecologic oncology team at a single institute conducted all procedures, and a dedicated radiologist at the same institute reviewed all data from imaging studies (e.g., magnetic resonance imaging and computed tomography). From 2011, neoadjuvant chemotherapy followed by interval debulking surgery was introduced in our institution, and 39 out of the 116 patients received this treatment. All 116 patients were treated with platinum based chemotherapy. These patients were analyzed for mutation type and clinical features including family history, personal breast cancer history, progression-free survival (PFS), OS, and response rate.

The response rate was determined using the Response Evaluation Criteria in Solid Tumors system. Specifically, we analyzed PFS and OS in patients with BRCA1/2 mutation and compared these data with those of patients with $B R C A 1 / 2$ wild type and VUS. PFS was defined as the period in months between the dates of diagnosis and relapse or last contact. OS was defined as the period in months between the dates of diagnosis and death or last contact.

\section{Direct sequencing}

Genetic testing for $B R C A 1$ and $B R C A 2$ (accession numbers NM_007294 and NM_000059, respectively) mutations was performed using direct sequencing as previously described [12]. The genetic mutations analyzed were confined to deleterious mutations such as frameshift or nonsense mutations. Variations were described following the nomenclature system of the Human Genome Variation Society (http://www. 
Table 2. Overall response rates after first chemotherapy in BRCA1/2-positive and sporadic epithelial ovarian cancer patients

\begin{tabular}{lccc} 
& No. of patients & Complete and partial response & p-value \\
BRCA1/2 mutation & 37 & $31(83.8)$ & $0.898^{\text {a) }}$ \\
BRCA1/2 VUS & 25 & $22(88.0)$ & $46(85.2)$ \\
BRCA1/2 wild type & 54 & $42)$ & \\
\hline
\end{tabular}

Values are presented as number (\%). VUS, variant of uncertain significance. ${ }^{\text {a)}}$ Chi-square Pearson's test.

hgvs.org/mutnomen) and the conventional nomenclature system from the Breast Cancer Information Core (BIC; http:// research.nhgri.nih.gov/bic/).

\section{Statistical analysis}

Statistical analysis was performed using IBM SPSS ver. 20.0 (IBM Corp., Armonk, NY). A Kolmogorov-Smirnov test was used to verify standard normal distributional assumptions. Patient clinical features including response rate, PFS, and OS were analyzed using an ANOVA test, a Pearson's chi-squared test, and Kaplan-Meier survival analysis. A p-value of less than 0.05 was regarded as statistically significant.

\section{Results}

\section{Patient characteristics}

Patient characteristics are shown in Table 1. Among the 116 EOC patients who underwent $B R C A 1 / 2$ gene tests by the polymerase chain reaction-denaturing high performance liquid chromatography-sequencing method, 37 (37/116, 31.9\%) $B R C A 1 / 2$ mutations were identified (BRCA1, 30; BRCA2, 7). In addition, 25 patients with $B R C A 1 / 2$ VUSs were identified $(25 / 116,21.6 \%)$ and two different types of BRCA1/2 VUS were found simultaneously in one patient.

No significant differences were detected in terms of mean age, International Federation of Gynecology and Obstetrics stage, cancer histology, grade, and performance of optimal surgery. Personal histories of breast cancer were observed in $48.6 \%$ of patients with BRCA1/2 mutation (18/37), $16.0 \%$ of patients with $B R C A 1 / 2$ VUS (4/25), and $7.4 \%$ of those with wild type $(4 / 54)(\mathrm{p}<0.001)$. A family history of BRCA1/2associated cancer was present in $48.6 \%$ of patients with BRCA1/2 mutation (18/37), 36.0\% of those with BRCA1/2 VUS $(9 / 25)$, and $37 \%$ of $B R C A 1 / 2$ patients with BRCA wild type $(20 / 54)(p=0.299)$.

\section{Response rates and survival}

The response rate was $83.8 \%$ in patients with $B R C A 1 / 2$ mutation (31/37), 88.0\% in patients with BRCA1/2 VUS $(22 / 25)$, and $85.2 \%$ in patients with $B R C A 1 / 2$ wild type $(46 / 54)$. No significant differences were detected between the three groups ( $\mathrm{p}=0.898$ ) (Table 2).

The median PFS was 17, 14, and 13 months for patients with $B R C A 1 / 2$ mutation, VUS, and wild type, respectively. Patients with $B R C A 1 / 2$ mutation had longer PFS than those with $B R C A 1 / 2$ wild type, although this difference did not achieve statistical significance $(p=0.071)$. No significant differences were detected in terms of PFS between patients with $B R C A 1 / 2$ VUS and BRCA1/2 mutation or wild type $(\mathrm{p}=0.772$ and $\mathrm{p}=0.455$, respectively) (Fig. 2).

The median OS was 33, 24, and 17 months in patients with BRCA1/2 mutation, VUS, and wild type, respectively. Patients with $B R C A 1 / 2$ mutation showed longer OS than those with $B R C A 1 / 2$ wild type $(\mathrm{p}=0.005)$. No significant differences were detected in OS between patients with $B R C A 1 / 2$ VUS and $B R C A 1 / 2$ mutation or wild type $(\mathrm{p}=0.459$ and $\mathrm{p}=0.211$, respectively) (Fig. 3).

\section{Frequently observed $B R C A 1 / 2$ alterations}

Frequently observed $B R C A 1 / 2$ alterations in this study are presented in Tables 3 and 4. The c.3627_3628insA (p.Leu1209 _Glu1210?fs) alteration in BRCA1 (exon 11) was recurrent in five patients $(5 / 37,13.5 \%)$. Among the 25 patients with BRCA1/2 VUS, c.8187G >T (p.Lys2729Asn) mutation in $B R C A 2$ (exon 18) was present in four $(4 / 25,16 \%$ ). All of the frequently observed alterations were reported in the Breast Cancer Information Core database.

\section{Discussion}

In the present study, we investigated the impact of $B R C A 1 / 2$ mutation status on the clinical features of EOC patients. Patients with BRCA1/2 mutation had improved OS 


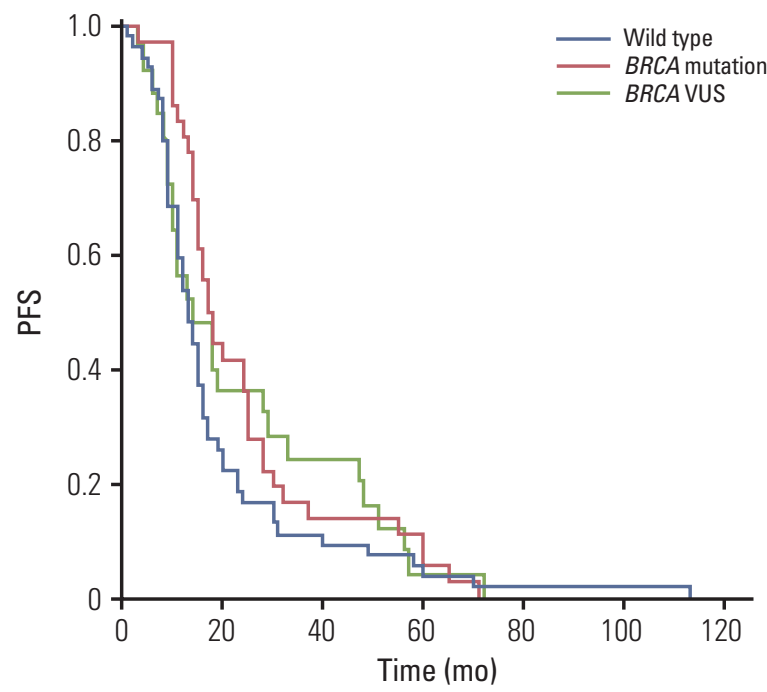

Fig. 2. Progression-free survival (PFS) curves according to $B R C A 1 / 2$ mutation status. Median PFS was 17 months for $B R C A 1 / 2$ mutation patients, 14 months for BRCA1/2 variant of uncertain significance (VUS) patients, and 13 months for BRCA1/2 wild type patients. A log-rank test revealed longer PFS for $B R C A 1 / 2$ mutation than wild type patients; however, this was not statistically significant $(\mathrm{p}=0.071)$. No differences were detected between $B R C A 1 / 2$ wild type and VUS ( $\mathrm{p}=0.455)$ or $B R C A 1 / 2$ mutation and VUS ( $\mathrm{p}=0.772)$.

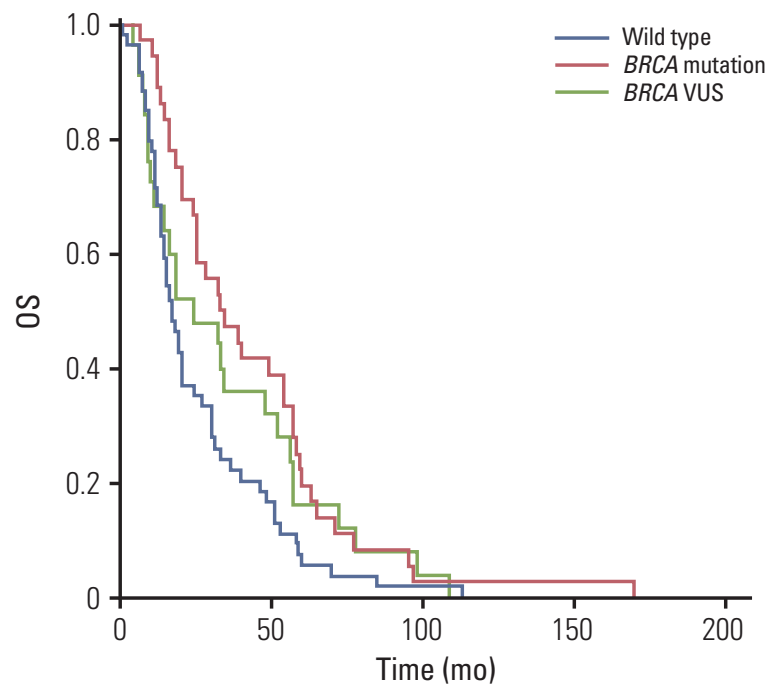

Fig. 3. Overall survival (OS) curves according to $B R C A 1 / 2$ mutation status. Median OS was 33 months for $B R C A 1 / 2$ mutation patients, 24 months for BRCA1/2 variant of uncertain significance (VUS) patients, and 17 months for $B R C A 1 / 2$ wild type patients. A log-rank test revealed significantly longer OS for $B R C A 1 / 2$ mutation than wild type patients $(\mathrm{p}=0.005)$. No differences were detected between $B R C A 1 / 2$ wild type and VUS $(\mathrm{p}=0.211)$ or $B R C A 1 / 2$ mutation and VUS $(\mathrm{p}=0.459)$.

Table 3. Frequently observed $B R C A 1 / 2$ mutations

\begin{tabular}{llllll} 
Gene & Site & \multicolumn{1}{c}{ Mutation } & Mutation type & No. (\%) (n=37) & BIC data \\
BRCA1 & Exon 11 & c.3627_3628insA (p.Leu1209_Glu1210?fs) & Frameshift & $5(13.5)$ & Yes \\
BRCA1 & Exon 7 & c.390C>A (p.Tyr130Ter) & Nonsense & $3(8.1)$ & Yes \\
BRCA1 & Exon 10 & c.1399A>T (p.Lys467Ter) & Nonsense & $2(5.4)$ & Yes \\
BRCA1 & Exon 11 & c.4041_4042delAG (p.Arg1347_Gly1348ArgAsnfs) & Frameshift & $2(5.4)$ & Yes \\
BRCA1 & Exon 11 & c.3442delG (p.Glu1148Argfs) & Frameshift & $2(5.4)$ & Yes \\
BRCA2 & Exon 15 & c.7480C>T (p.Arg2494Ter) & Nonsense & $2(5.4)$ & Yes \\
\hline
\end{tabular}

BIC, Breast Cancer Information Core.

Table 4. Frequently observed BRCA1/2 VUSs

\begin{tabular}{lllllc} 
Gene & Site & \multicolumn{1}{c}{ Mutation } & Mutation type & No. $(\%)(\mathbf{n = 2 5})$ & BIC data \\
BRCA2 & Exon 18 & c.8187G $>$ T (p.Lys2729Asn) & Missense & $4(16.0)$ & Yes \\
BRCA1 & Exon 16 & c.5339T>C (p.Leu1780Pro) & Missense & $3(12.0)$ & Yes \\
BRCA1 & Exon 16 & c.4883T>C (p.Met1628Thr) & Missense & $2(8.0)$ & Yes \\
\hline
\end{tabular}

VUS, variant of uncertain significance; BIC, Breast Cancer Information Core. 
as compared to those with $B R C A 1 / 2$ wild type. However, no significant differences in PFS and response rates were detected between the groups included in this study. BRCA1/2 mutation and VUS patients had similar prognoses. Greater sensitivity to platinum-based chemotherapy among patients with $B R C A 1 / 2$ mutation was not identified in this study. To the best of our knowledge, this is the first study that compared the clinical outcomes of EOC patients with $B R C A 1 / 2$ mutation, VUS, and wild type.

A favorable prognosis for patients with $B R C A 1 / 2$ mutation over that of patients with $B R C A 1 / 2$ wild type was identified in this study, although no significant difference was detected in PFS. The underlying mechanism of BRCA1/2 mutation conferring a favorable prognosis remains unclear. The main function of $B R C A 1 / 2$ proteins is to promote DNA doublestrand break repair via homologous recombination. $B R C A 1$ has been implicated in many cellular functions, including DNA repair, the maintenance of genomic integrity, and cell cycle checkpoint control $[17,18]$. The main function of $B R C A 2$ appears to involve interaction with RAD51 during homologous recombination DNA repair [19]. Cells with mutated $B R C A 1 / 2$ proteins may therefore be rendered less capable of repairing chemotherapy-induced DNA damage, potentially leading to an improved response to treatment. This is known as 'synthetic lethality,' i.e., the enhanced lethality of DNAdamaging agents.

Our data failed to show significantly longer PFS or a better response rate to chemotherapy in patients with $B R C A 1 / 2$ mutation over those of patients with $B R C A 1 / 2$ VUS or wild type. To explain the discrepancy between our data and those of prior studies, a more complex model is necessary to clarify specific mechanisms of $B R C A 1 / 2$ dysfunction that result in better outcomes for EOC patients. It is estimated that approximately $50 \%$ of sporadic EOCs show BRCA1 or BRCA2 dysfunction through different mechanisms. Tumors that share molecular features of BRCA1/2-mutant tumors (i.e., BRCAness) could also emerge in this process that affects or is affected by normal $B R C A 1 / 2$ gene function $[20,21]$. Previous studies reported that low BRCA1 or BRCA2 expression in sporadic EOC could confer similar effects on prognosis as $B R C A 1$ or $B R C A 2$ mutation. Specifically, low BRCA1 expression measured by reverse transcription polymerase chain reaction was shown to be a positive prognostic factor for both OS and PFS in patients with sporadic EOCs [22]. This finding indicates that low BRCA1 expression status in sporadic EOC has a similar impact on prognosis as germline $B R C A 1 / 2$ mutation. Therefore, if "BRCAness" could be measured quantitatively in our study, a precise comparison between "BRCAness" positive and negative groups would possible.

Interpretation of the clinical implications of $B R C A 1 / 2$ VUS remains challenging because misperception by a physician regarding the implications of VUS could lead to inappropri- ate risk-reducing surgery, neglect, or providing misinformation to patients. Myriad Genetic Laboratories (Salt Lake City, UT) reported that about $7 \%$ of their molecular diagnoses of hereditary breast and ovarian cancer are linked to VUSs $[23,24]$, and these alterations have been identified more commonly in African-American than in Hispanic populations $[25,26]$. However, few reports have analyzed VUS prevalence in Asian patients. Additionally, limited information is available regarding the clinical features of EOC patients with $B R C A 1 / 2$ VUS.

Despite the possibility of selection bias, $21.6 \%$ of patients $(25 / 116)$ who underwent BRCA1/2 genetic tests were found to have BRCA1/2 VUS. Among patients with BRCA1/2 VUS, $16 \%(4 / 25)$ had a personal history of double primary breast cancer and 36\% (9/25) had a family history of breast or ovarian cancer in first-degree relatives. One of the VUSs found in this study (BRCA1 c.5339T $>$ C) is highly suspected to be a deleterious mutation based on the patients' family histories of $B R C A 1 / 2$-associated cancer, personal histories of breast cancer, and population frequency.

Identification of founder mutations is required to improve the quality of genetic counseling. Moreover, using a more specific approach to molecular testing leads to greater costeffectiveness. If we can recognize differences in susceptibility due to a specific founder mutation, it will be possible to define the role of risk reducing surgery. Frequently observed alterations in this study are presented in Tables 3 and 4 . Studies to identify founder mutations have been conducted in Asian countries [27], and one report analyzed possible candidates of a founder mutation in Korea [13]. However, specific mutations that account for a high frequency of cases, such as that observed in the Ashkenazi Jewish population, have not yet been discovered. The frequently observed $B R C A 1 / 2$ alterations found in this study were not identified in a previous study conducted in Korea. Accordingly, an investigation including a larger number of cases must be analyzed to provide accurate information regarding the frequency of founder mutations.

It should be noted that this study had several limitations. Specifically, it included a small number of patients with $B R C A 1 / 2$ mutations because of the low rate of genetic testing, had a retrospective design with the possibility of selection bias, and a short follow-up period. Moreover, 595 patients declined BRCA1/2 genetic testing. Conversely, a previous report showed few barriers to participating in genetic counseling and BRCA1/2 testing in Western countries, with a testing rate of $81 \%$ [28]. The low rate of genetic testing $(16.3 \%$, $116 / 711$ ) in the present study may be the result of low public awareness regarding its availability. In addition, this study included patients representative of a cancer center population, and all study participants had been affected by EOC. Thus, our findings may not apply to patients who have not 
had cancer and are undergoing genetic testing due to family history alone.

Routine tests for $B R C A 1 / 2$ germline mutation status in patients with EOC may be warranted, as it has been demonstrated that a deficiency in the BRCA1/2 gene confers substantial sensitivity to a chemotherapeutic agent, namely poly(ADP-ribose) polymerase-1 (PARP) inhibitor (olaparib) [29]. Further research is required to determine whether application of this agent to EOCs with pathologic $B R C A 1 / 2$ VUS is beneficial or not. Some patients with $B R C A 1 / 2$ VUS may be responsive to treatment with PARP inhibitors, which results in synthetic lethality of cells that have deficient homologous recombination or double-strand DNA repair. This might improve survival among such patients. Further prospective cohort studies with longer follow-up periods as well as "BRCAness" quantification are needed to enable a precise understanding of the role of BRCAness on the clinical features of EOC patients.

\section{Conclusion}

Our study provides useful data for counseling EOC patients with BRCA1/2 mutation, VUS, and wild type. Patients with $B R C A 1 / 2$ mutation had more favorable prognosis, or significantly longer OS than those with $B R C A 1 / 2$ wild type, while they have similar prognoses as patients with $B R C A 1 / 2$ VUS.

\section{Conflicts of Interest}

Conflict of interest relevant to this article was not reported.

\section{Acknowledgments}

This research was supported by the Basic Science Research Program through the National Research Foundation of Korea (NRF) funded by the Ministry of Science, ICT \& Future Planning (2014R1A1A1A05002926; 2015R1A2A2A01008162; 2015R1C1A2A01053516).

\section{References}

1. Kim SI, Lim MC, Lim J, Won YJ, Seo SS, Kang S, et al. Incidence of epithelial ovarian cancer according to histologic subtypes in Korea, 1999 to 2012. J Gynecol Oncol. 2016;27:e5.

2. Ramus SJ, Gayther SA. The contribution of BRCA1 and BRCA2 to ovarian cancer. Mol Oncol. 2009;3:138-50.

3. Chen S, Iversen ES, Friebel T, Finkelstein D, Weber BL, Eisen A, et al. Characterization of BRCA1 and BRCA2 mutations in a large United States sample. J Clin Oncol. 2006;24:863-71.

4. Yang D, Khan S, Sun Y, Hess K, Shmulevich I, Sood AK, et al. Association of BRCA1 and BRCA2 mutations with survival, chemotherapy sensitivity, and gene mutator phenotype in patients with ovarian cancer. JAMA. 2011;306:1557-65.

5. Sun C, Li N, Ding D, Weng D, Meng L, Chen G, et al. The role of BRCA status on the prognosis of patients with epithelial ovarian cancer: a systematic review of the literature with a meta-analysis. PLoS One. 2014;9:e95285.

6. Levine DA, Federici MG, Reuter VE, Boyd J. Cell proliferation and apoptosis in BRCA-associated hereditary ovarian cancer. Gynecol Oncol. 2002;85:431-4.

7. McLaughlin JR, Rosen B, Moody J, Pal T, Fan I, Shaw PA, et al. Long-term ovarian cancer survival associated with mutation in BRCA1 or BRCA2. J Natl Cancer Inst. 2013;105:141-8.

8. Kotsopoulos J, Rosen B, Fan I, Moody J, McLaughlin JR, Risch $\mathrm{H}$, et al. Ten-year survival after epithelial ovarian cancer is not associated with BRCA mutation status. Gynecol Oncol. 2016;140:42-7.

9. Johannsson OT, Ranstam J, Borg A, Olsson H. Survival of BRCA1 breast and ovarian cancer patients: a population-based study from southern Sweden. J Clin Oncol. 1998;16:397-404.

10. Pharoah PD, Easton DF, Stockton DL, Gayther S, Ponder BA. Survival in familial, BRCA1-associated, and BRCA2-associated epithelial ovarian cancer. United Kingdom Coordinating Committee for Cancer Research (UKCCCR) Familial Ovarian Cancer Study Group. Cancer Res. 1999;59:868-71.

11. Lim MC, Kang S, Seo SS, Kong SY, Lee BY, Lee SK, et al. BRCA1 and BRCA2 germline mutations in Korean ovarian cancer patients. J Cancer Res Clin Oncol. 2009;135:1593-9.

12. Kim YT, Nam EJ, Yoon BS, Kim SW, Kim SH, Kim JH, et al. Germline mutations of BRCA1 and BRCA2 in Korean sporadic ovarian carcinoma. Gynecol Oncol. 2005;99:585-90. 
13. Choi MC, Heo JH, Jang JH, Jung SG, Park H, Joo WD, et al. Germline mutations of BRCA1 and BRCA2 in Korean ovarian cancer patients: finding founder mutations. Int J Gynecol Cancer. 2015;25:1386-91.

14. Kang E, Kim SW. The Korean hereditary breast cancer study: review and future perspectives. J Breast Cancer. 2013;16:24553.

15. Aida H, Takakuwa K, Nagata H, Tsuneki I, Takano M, Tsuji $\mathrm{S}$, et al. Clinical features of ovarian cancer in Japanese women with germ-line mutations of BRCA1. Clin Cancer Res. 1998;4: 235-40.

16. Calo V, Bruno L, La Paglia L, Perez M, Margarese N, Di Gaudio F, et al. The clinical significance of unknown sequence variants in BRCA genes. Cancers (Basel). 2010;2:1644-60.

17. Xu X, Weaver Z, Linke SP, Li C, Gotay J, Wang XW, et al. Centrosome amplification and a defective G2-M cell cycle checkpoint induce genetic instability in BRCA1 exon 11 isoformdeficient cells. Mol Cell. 1999;3:389-95.

18. Yang H, Jeffrey PD, Miller J, Kinnucan E, Sun Y, Thoma NH, et al. BRCA2 function in DNA binding and recombination from a BRCA2-DSS1-ssDNA structure. Science. 2002;297:183748.

19. Gudmundsdottir K, Ashworth A. The roles of BRCA1 and BRCA2 and associated proteins in the maintenance of genomic stability. Oncogene. 2006;25:5864-74.

20. Muggia F, Safra T. 'BRCAness' and its implications for platinum action in gynecologic cancer. Anticancer Res. 2014;34: 551-6.

21. Lord CJ, Ashworth A. BRCAness revisited. Nat Rev Cancer. 2016;16:110-20.

22. Quinn JE, James CR, Stewart GE, Mulligan JM, White P, Chang GK, et al. BRCA1 mRNA expression levels predict for overall survival in ovarian cancer after chemotherapy. Clin Cancer Res. 2007;13:7413-20.

23. Frank TS, Deffenbaugh AM, Reid JE, Hulick M, Ward BE, Lingenfelter B, et al. Clinical characteristics of individuals with germline mutations in BRCA1 and BRCA2: analysis of 10,000 individuals. J Clin Oncol. 2002;20:1480-90.

24. Easton DF, Deffenbaugh AM, Pruss D, Frye C, Wenstrup RJ, Allen-Brady K, et al. A systematic genetic assessment of 1,433 sequence variants of unknown clinical significance in the BRCA1 and BRCA2 breast cancer-predisposition genes. Am J Hum Genet. 2007;81:873-83.

25. Nanda R, Schumm LP, Cummings S, Fackenthal JD, Sveen L, Ademuyiwa F, et al. Genetic testing in an ethnically diverse cohort of high-risk women: a comparative analysis of BRCA1 and BRCA2 mutations in American families of European and African ancestry. JAMA. 2005;294:1925-33.

26. Weitzel JN, Lagos V, Blazer KR, Nelson R, Ricker C, Herzog J, et al. Prevalence of BRCA mutations and founder effect in high-risk Hispanic families. Cancer Epidemiol Biomarkers Prev. 2005;14:1666-71.

27. Sekine M, Nagata H, Tsuji S, Hirai Y, Fujimoto S, Hatae M, et al. Mutational analysis of BRCA1 and BRCA2 and clinicopathologic analysis of ovarian cancer in 82 ovarian cancer families: two common founder mutations of BRCA1 in Japanese population. Clin Cancer Res. 2001;7:3144-50.

28. Schlich-Bakker KJ, ten Kroode HF, Warlam-Rodenhuis CC, van den Bout J, Ausems MG. Barriers to participating in genetic counseling and BRCA testing during primary treatment for breast cancer. Genet Med. 2007;9:766-77.

29. Fong PC, Boss DS, Yap TA, Tutt A, Wu P, Mergui-Roelvink $\mathrm{M}$, et al. Inhibition of poly(ADP-ribose) polymerase in tumors from BRCA mutation carriers. N Engl J Med. 2009;361:123-34. 